Supporting Information

\title{
Heterogeneous Sensitization of Metal-Organic Framework Driven Metal@Metal Oxide Complex Catalysts on an Oxide Nanofiber Scaffold Toward Superior Gas Sensors
}

Won-Tae Koo, ${ }^{\dagger}$ Seon-Jin Choi, ${ }^{\dagger, \S}$ Sang-Joon Kim, ${ }^{\dagger} \mathrm{Ji}^{-S o o}$ Jang, ${ }^{\dagger}$ Harry L. Tuller, ${ }^{\ddagger}$ and IlDoo Kim*, ${ }^{*}$

${ }^{\dagger}$ Department of Materials Science and Engineering and ${ }^{\S}$ Applied Science Research Institute, Korea Advanced Institute of Science and Technology (KAIST), 291 Daehak-ro, Yuseong-gu, Daejeon 305-701, Korea

${ }^{\ddagger}$ Department of Materials Science and Engineering, Massachusetts Institute of Technology Cambridge, MA, 02139, United States

\section{Contents}

1. Experimental details

2. SEM image of ZIF-8 and Pd@ZIF-8

3. Characterization of ZIF-8 and Pd@ZIF-8

4. EDS elemental mapping of AMH/PVP/Pd@ZIF-8 NFs

5. SEM image of $\mathrm{ZnO}-\mathrm{WO}_{3} \mathrm{NFs}$, as-spun $\mathrm{AMH} / \mathrm{PVP} \mathrm{NFs}$, and $\mathrm{WO}_{3} \mathrm{NFs}$

6. EDS elemental mapping of Pd@ZnO-WO $\mathrm{WO}_{3} \mathrm{NFs}$

7. PXRD analysis

8. TEM image of Pd(polyol) and SEM image of Pd(polyol)-WO3 NFs, Pd(polyol)-ZnO$\mathrm{WO}_{3} \mathrm{NFs}$

9. Supplementary sensing characteristics

10. Cyclic sensing response

11. UPS analysis

12. $\mathrm{N}_{2}$ adsorption/desorption and pore size distribution

13. Supporting table

14. Supporting references 


\section{Experimental details}

Materials: Polyvinylpyrrolidone (PVP, $\mathrm{M}_{\mathrm{w}} \sim 1,300,000 \mathrm{~g} / \mathrm{mol}$ ), 2-methylimidazole (Hmin, 99.0\%), sodium borohydride $\left(\mathrm{NaBH}_{4}, 96 \%\right)$, and palladium chloride $\left(\mathrm{PdCl}_{2}\right)$ were purchased from Aldrich. Zinc nitrate hexahydrate $\left(\left[\mathrm{Zn}\left(\mathrm{NO}_{3}\right)_{2} \cdot 6 \mathrm{H}_{2} \mathrm{O}\right], 98 \%\right)$, ammonium metatungstate hydrate (AMH, [( $\left.\left.\left(\mathrm{NH}_{4}\right)_{6} \mathrm{H}_{2} \mathrm{~W}_{12} \mathrm{O}_{40} \times \mathrm{xH}_{2} \mathrm{O}\right]\right)$, methanol (MeOH, 99.9\%), ethylene glycol (EG, 99.8\%), acetone (99.9\%), and potassium tetrachloropalladate(II) $\left(\mathrm{K}_{2} \mathrm{PdCl}_{4}\right)$ were purchased from Sigma-Aldrich. All chemicals were used without further purification.

Synthesis of pristine $\mathbf{W O}_{3}$ nanofibers (pristine $\mathbf{W O}_{3} \mathbf{N F s}$ ): Pristine $\mathrm{WO}_{3} \mathrm{NFs}$ were prepared by electrospinning, followed by a calcination step. The electrospinning solution, composed of $0.4 \mathrm{~g}$ of AMH and $0.5 \mathrm{~g}$ of PVP dissolved in deionized water (DI-water), was continuously stirred at room temperature for $5 \mathrm{~h}$. Electrospinning was conducted with the prepared solution at a feeding rate of 0.1 $\mathrm{mL} / \mathrm{min}$ using a syringe pump at $25^{\circ} \mathrm{C}$ within an environment with $30 \% \mathrm{RH}$. During electrospinning, a voltage of $16 \mathrm{kV}$ and a distance of $15 \mathrm{~cm}$ were maintained between the tip of the syringe needle (21 gauge) and the stainless steel foil collector. The as-spun AMH/PVP composite NFs were calcined at $500{ }^{\circ} \mathrm{C}$ for $1 \mathrm{~h}$ in air atmosphere with a temperture ramping rate of $4{ }^{\circ} \mathrm{C} / \mathrm{min}$.

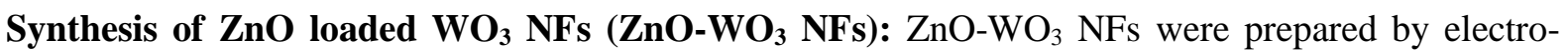
spinning, followed by a calcination step. The electrospinning solution, was composed of $0.4 \mathrm{~g}$ of AMH and $0.5 \mathrm{~g}$ of PVP dissolved in DI-water solution. The solution was stirred at room temperature for $5 \mathrm{~h}$ following the addition of ZIF-8 to the solution. The same electrospinning and calcination conditions were used as described above for synthesis of pristine $\mathrm{WO}_{3}$ nanofibers.

Synthesis of Pd nanoparticles (NPs) by polyol process (Pd(polyol)): $0.5 \mathrm{~g}$ of $\mathrm{PdCl}_{2}$ dissolved EG solution (5 mL) was injected into a $500 \mathrm{~mL}$ flask containing $40 \mathrm{~mL}$ of EG in a heated oil bath $\left(150^{\circ} \mathrm{C}\right)$. Then, $0.5 \mathrm{~g}$ of PVP dissolved EG solution $(20 \mathrm{~mL})$ was added to the flask. After $1 \mathrm{~h}, 25 \mathrm{~mL}$ of acetone was added into the EG solution. The mixed solution was centrifuged at $3000 \mathrm{rpm}$ for 10 min and washed five times with DI-water water. The Pd NPs synthesized by the polyol process were dispersed in $3 \mathrm{~mL}$ of DI-water.

Synthesis of Pd(polyol) $\mathrm{NPs}$ loaded $\mathrm{WO}_{3}$ and $\mathrm{ZnO}-\mathrm{WO}_{3} \mathrm{NFs}$ (Pd(polyol)- $\mathrm{WO}_{3} \mathrm{NFs}$ and Pd(polyol)-ZnO-WO $\mathbf{W}_{3}$ NFs): $\mathrm{Pd}\left(\right.$ polyol)- $\mathrm{WO}_{3}$ NFs and $\mathrm{Pd}\left(\right.$ polyol)-ZnO- $\mathrm{WO}_{3}$ NFs were prepared by electrospinning and calcination steps. The electrospinning solution was composed initially of $0.4 \mathrm{~g}$ of $\mathrm{AMH}$ and $0.5 \mathrm{~g}$ of PVP dissolved in DI-water solution to which a $300 \mathrm{~mL}$ Pd(polyol) suspension was 
added. To synthesize Pd(polyol)-ZnO- $\mathrm{WO}_{3} \mathrm{NFs}, 0.5 \mathrm{mg}$ of ZIF-8 was also added into the electrospinning solution. Then, the electrospinning solution was continuously stirred at room temperature for $5 \mathrm{~h}$. The same electrospinning and calcination conditions were used as described above for synthesis of pristine $\mathrm{WO}_{3} \mathrm{NFs}$.

Sensor fabrication: To investigate the sensor characteristics of pristine $\mathrm{WO}_{3} \mathrm{NFs}, \mathrm{ZnO}-\mathrm{WO}_{3} \mathrm{NFs}$, Pd(polyol)- $\mathrm{WO}_{3}$ NFs, Pd(polyol)-ZnO-WO $\mathrm{WO}_{3} \mathrm{NFs}$, and Pd@ZnO-WO 3 NFs, three different sensing materials were dispersed in ethanol and then the solutions were drop-coated onto the three sensor substrates (area: $2.5 \mathrm{~mm}$ x $2.5 \mathrm{~mm}$, thickness $0.2 \mathrm{~mm}$ ), respectively. The alumina sensor substrate was printed with two parallel Au electrodes (width: $25 \mu \mathrm{m}$, gap size: $70 \mu \mathrm{m}$ ) on the top of the substrate and a Pt microheater on its back side.

Characterization: The microstructures and morphologies were analyzed by field emission scanning electron microscopy (FE-SEM, Nova230, FEI) and field emission transmission electron microscopy (FE-TEM, Tecnai G2 F30 S-Twin, FEI). The powder X-ray diffraction (PXRD, D/MAX-2500, Rigaku) analysis with $\mathrm{Cu} \mathrm{K} \alpha$ radiation $(\lambda=1.5418 \AA$ ) was carried out to analyze the crystal structures. The chemical bonding states were investigated using X-ray photoelectron spectroscopy (XPS, Sigma Probe, Thermo VG Scientific). $\mathrm{N}_{2}$ adsorption/desorption isotherms (Tristar 3020, Micromeritics) were conducted at $77 \mathrm{~K}$. Thermal stability was examined by thermal gravimetric analysis (TGA, Labsys Evo, Setaram). Inductively coupled plasma optical emission spectrometer (ICP-OES, iCAP 6300 Duo, Thermo Scientific) analysis was used to analyze the loading concentration of catalyst in the composite. Ultraviolet photoelectron spectroscopy (UPS) was conducted to analyze the work function of the samples. 
2. SEM image of ZIF-8 and Pd@ZIF-8

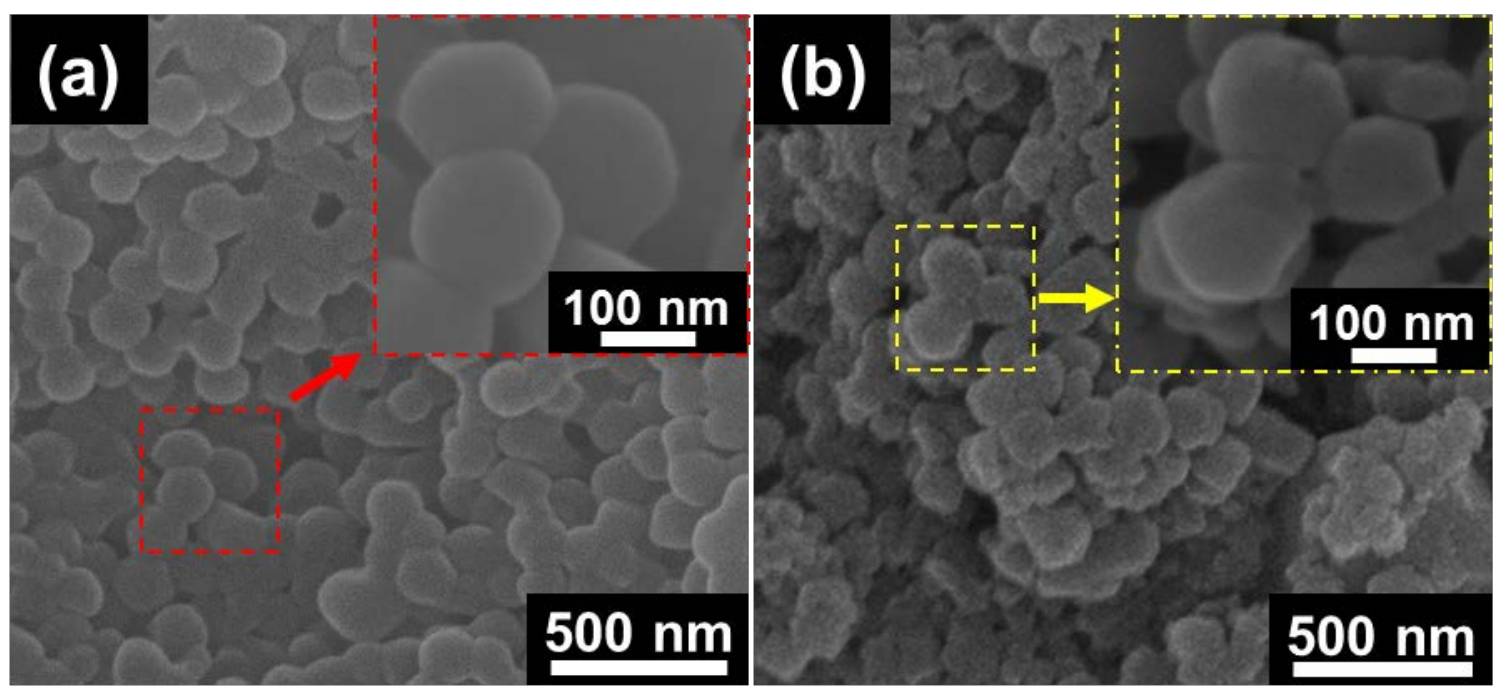

Figure S1. SEM images of: (a) ZIF-8, and (b) Pd@ZIF-8. 
3. Characterization of ZIF-8 and Pd@ZIF-8
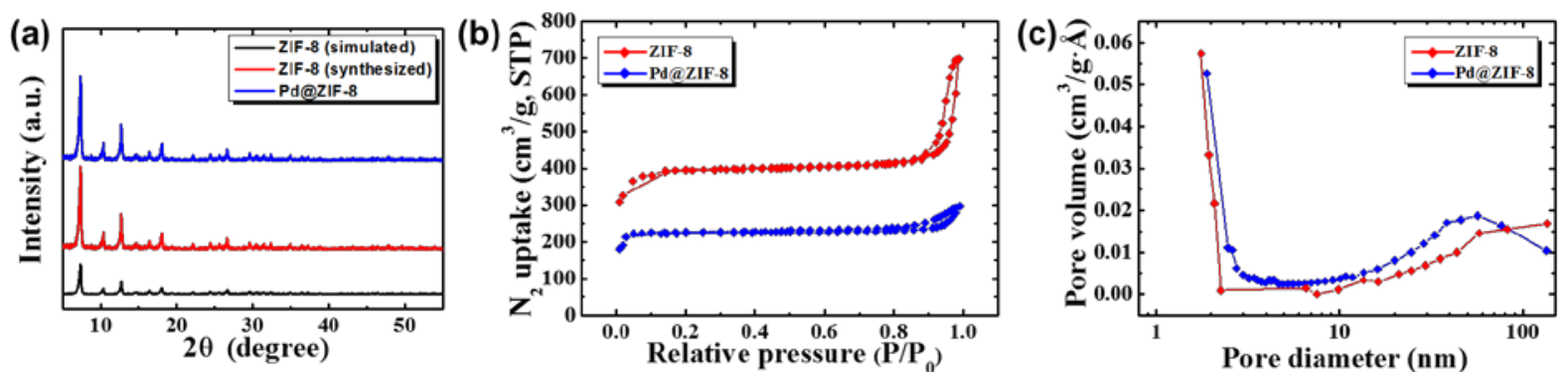

Figure S2. (a) PXRD analysis of ZIF-8 and Pd@ZIF-8, (b) $\mathrm{N}_{2}$ adsorption/desorption isotherms of assynthesized ZIF-8 and Pd@ZIF-8, and (c) Pore size distribution of ZIF-8 and Pd@ZIF-8. 


\section{EDS elemental mapping of AMH/PVP/Pd@ZIF-8 NFs}

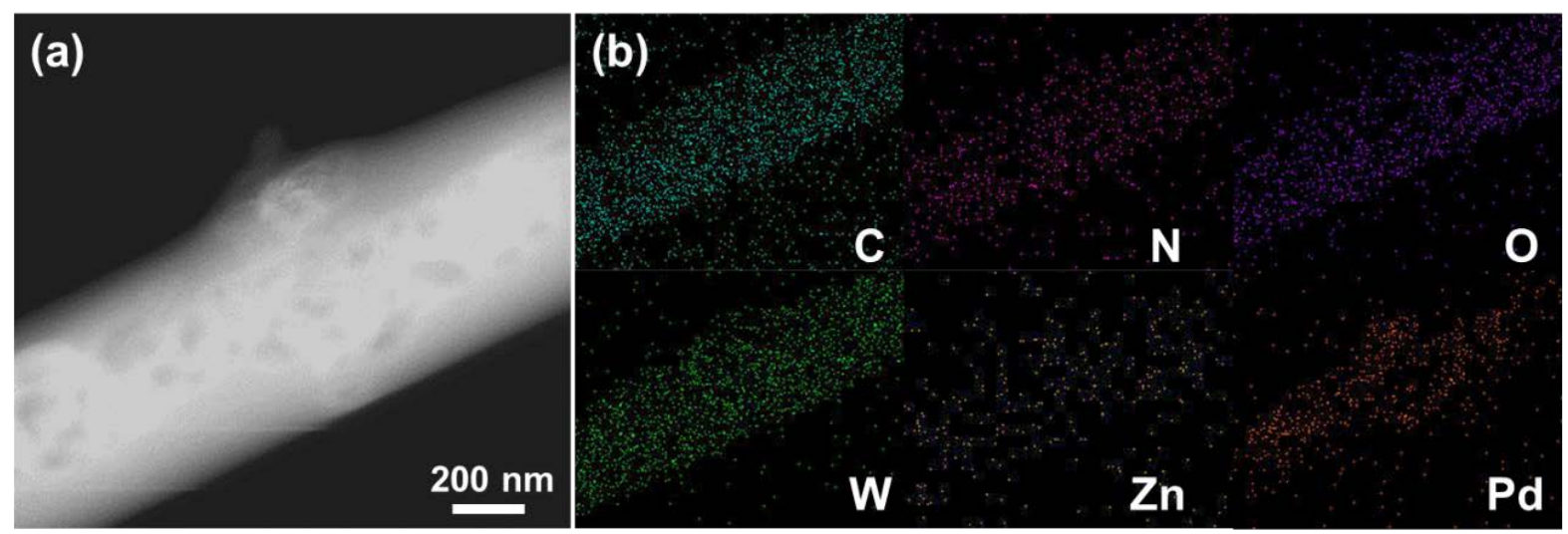

Figure S3. EDS elemental mapping of AMH/PVP/Pd@ZIF-8 NFs: (a) Scanning TEM (STEM) image, and (b) elemental mapping of C, N, O, W, Zn, and Pd 
5. SEM image of ZnO-WO $\mathrm{WH}_{3} \mathrm{NF}$, as-spun AMH/PVP NFs, and $\mathrm{WO}_{3} \mathrm{NFs}$

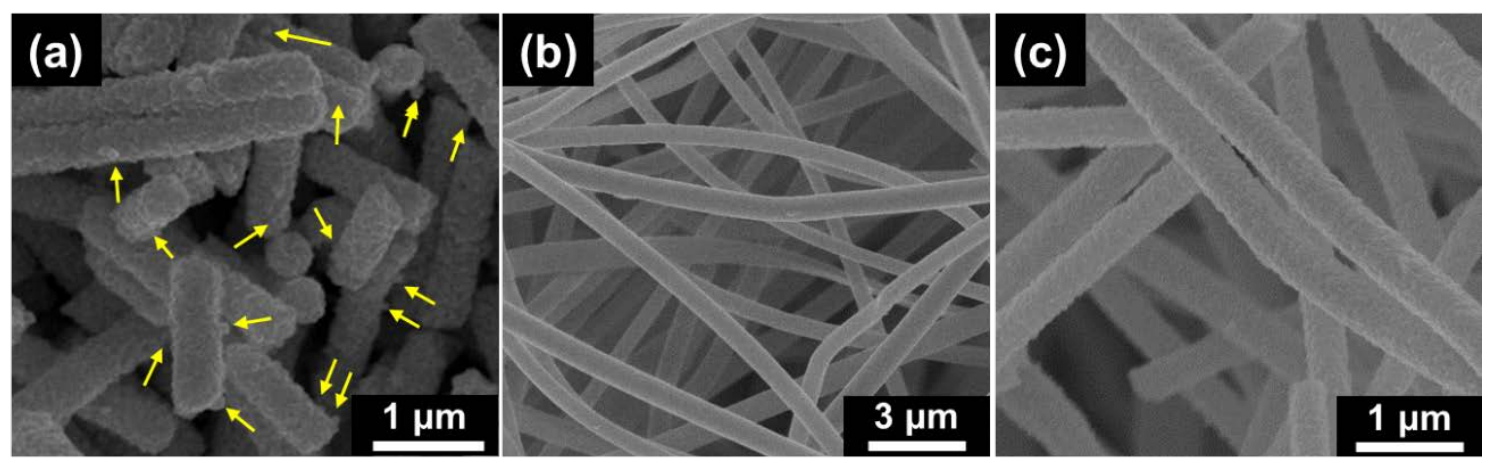

Figure S4. SEM images of: (a) ZnO-WO $\mathrm{W}_{3} \mathrm{NFs}$, (b) as-spun AMH/PVP composite NFs, and (c) $\mathrm{WO}_{3}$ NFs. 


\section{EDS elemental mapping of Pd@ZnO-WO3 NFs}

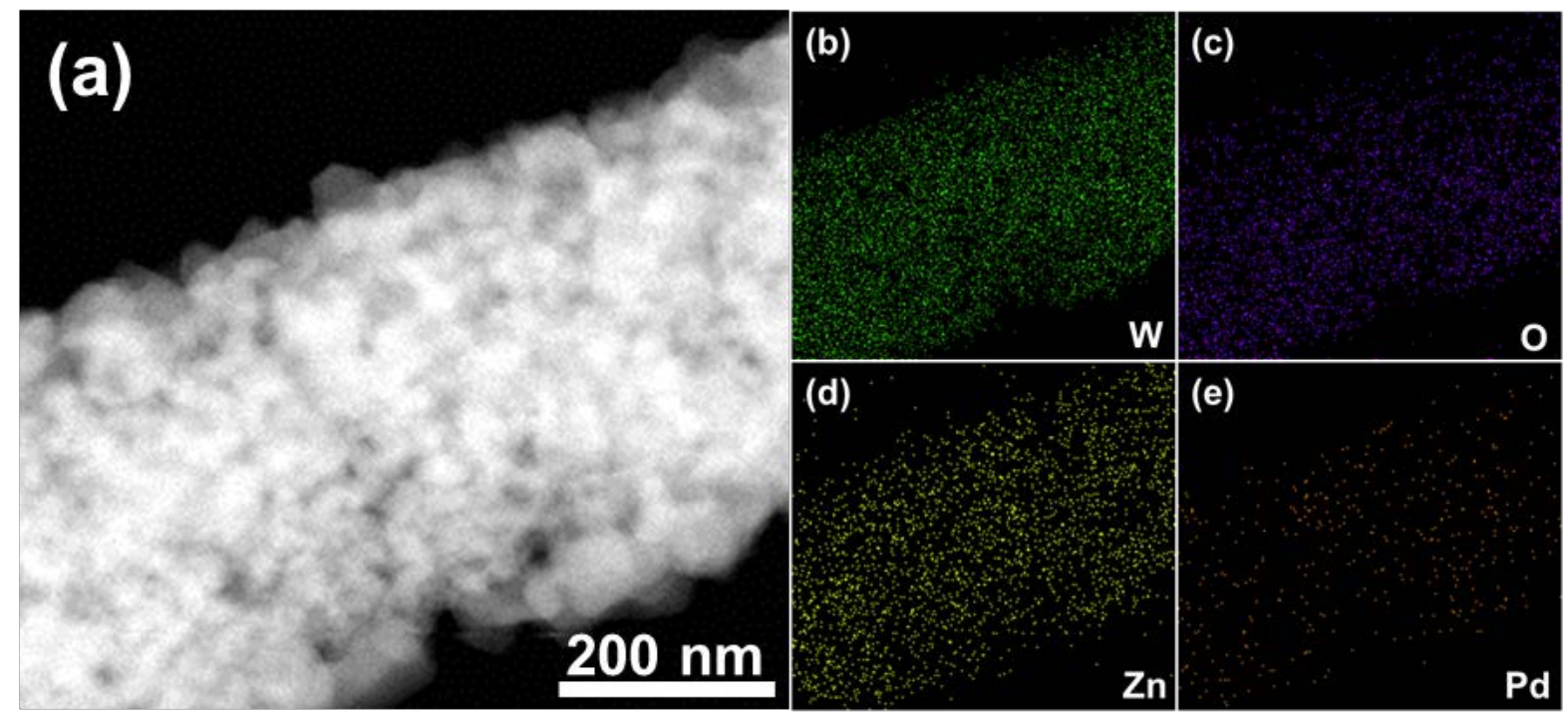

Figure S5. EDS elemental mapping of Pd@ZnO- $\mathrm{WO}_{3}$ NFs: (a) Scanning TEM (STEM) image, and elemental mapping of (b) W, (c) O, (d) Zn, and (e) Pd. 


\section{PXRD analysis}

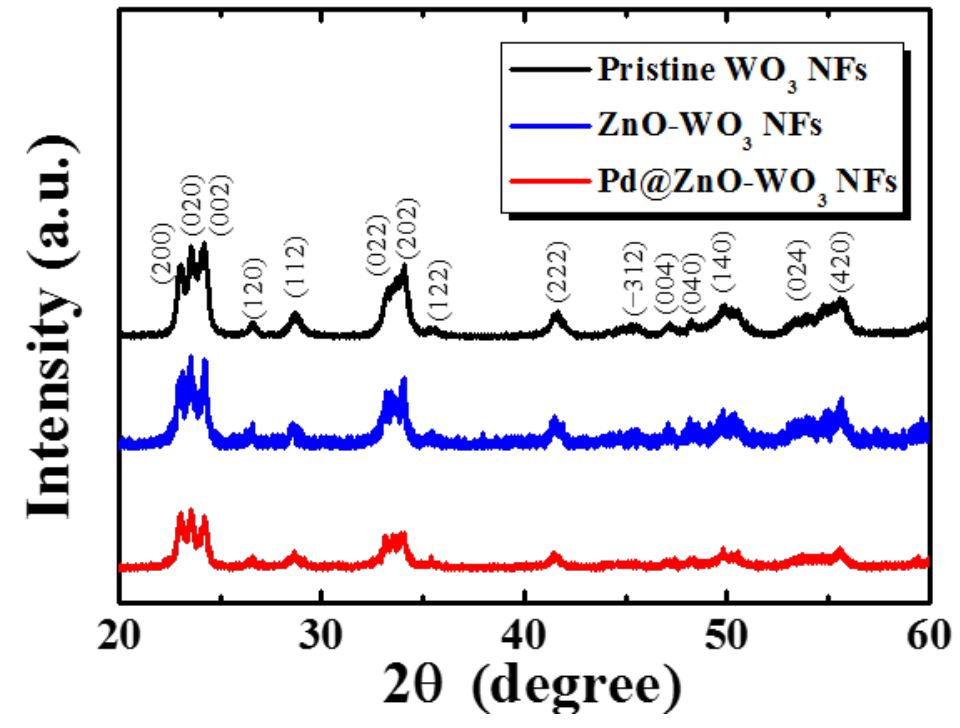

Figure S6. PXRD analysis of pristine $\mathrm{WO}_{3} \mathrm{NFs}, \mathrm{ZnO}-\mathrm{WO}_{3} \mathrm{NFs}$, and $\mathrm{Pd} @ \mathrm{ZnO}-\mathrm{WO}_{3} \mathrm{NFs}$. 
8. TEM image of Pd(polyol) and SEM image of Pd(polyol)-WO $\mathrm{WF}_{3} \mathrm{NF}$, Pd(polyol)$\mathrm{ZnO}-\mathrm{WO}_{3} \mathrm{NFs}$

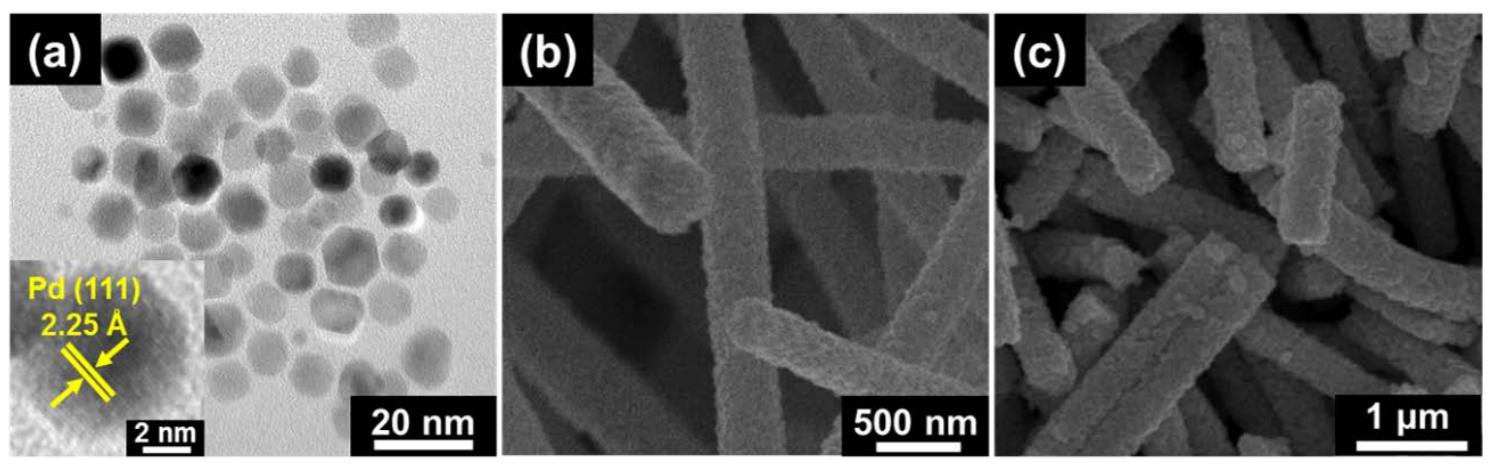

Figure S7. (a) TEM image of Pd(polyol) and SEM image of (b) Pd(polyol)-WO $\mathrm{W}_{3} \mathrm{NFs}$, (c) Pd(polyol)$\mathrm{ZnO}-\mathrm{WO}_{3} \mathrm{NFs}$. 


\section{Supplementary sensing characteristics}

(a)

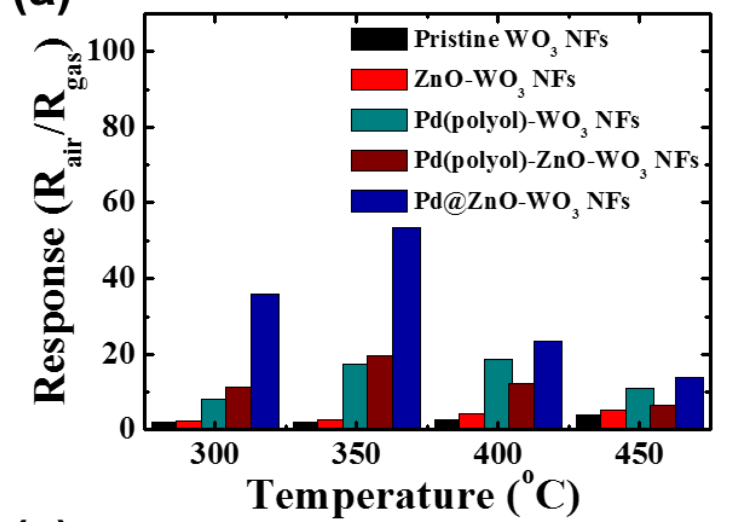

(c)

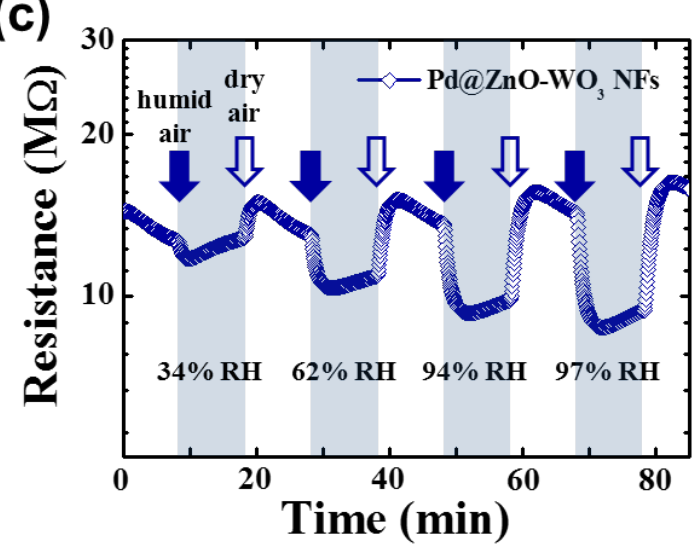

(b)

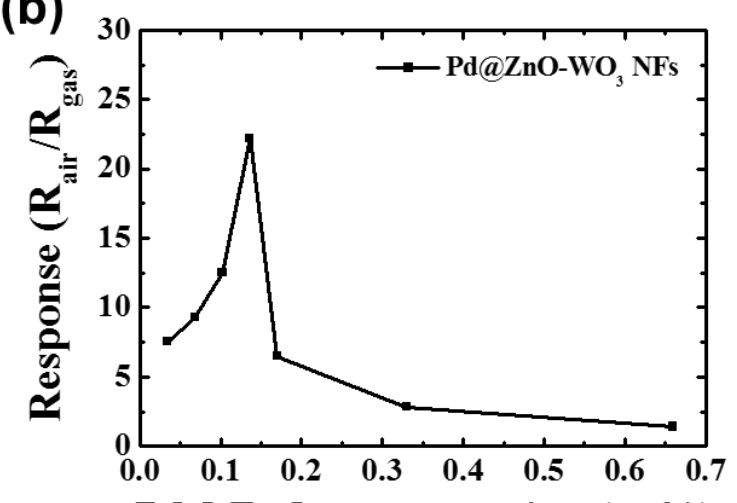

(d)

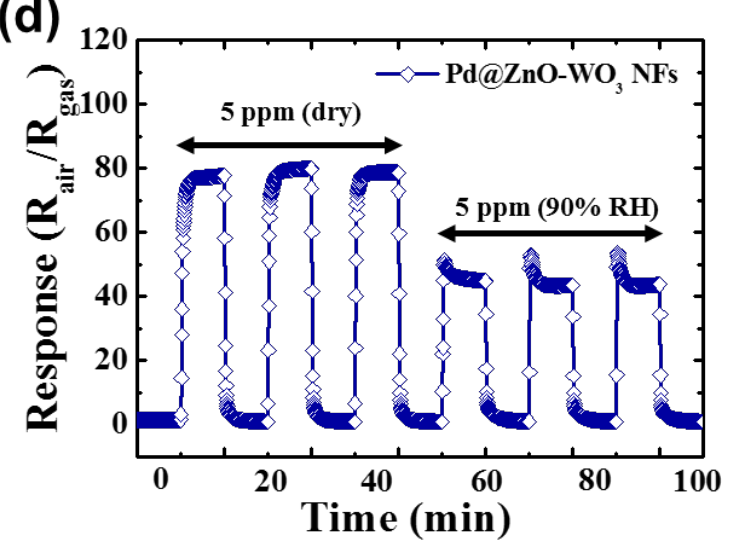

Figure S8. (a) Temperature dependent toluene response characteristics to $5 \mathrm{ppm}$ in a temperature range of 300-450 ${ }^{\circ} \mathrm{C}$, (b) Sensing characteristics of Pd@ZnO-WO $\mathrm{W}_{3} \mathrm{NFs}$ with the 1 ppm of toluene at $350{ }^{\circ} \mathrm{C}$ in different concentration of Pd@ZnO, (c) Dynamic resistance response of Pd@ZnO-WO NFs in different relative humidities at $350^{\circ} \mathrm{C}$, and (d) Response characteristics of Pd@ZnO-WO 3 NFs towards 5 ppm toluene at $350^{\circ} \mathrm{C}$ in dry and humid conditions. 
10. Cyclic sensing response

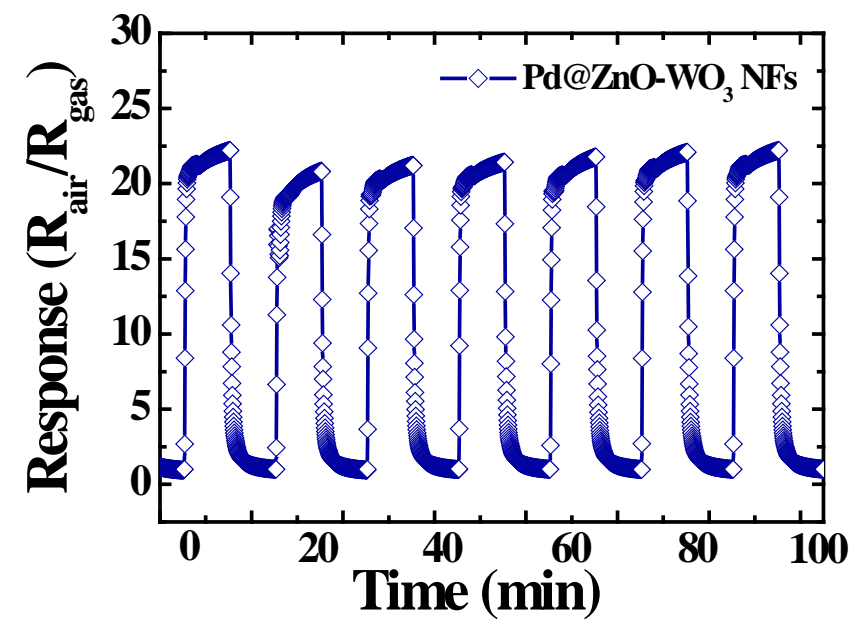

Figure S9. Cyclic sensing response of Pd@ZnO- $\mathrm{WO}_{3} \mathrm{NFs}$ toward toluene with gas concentration of 1 ppm at $350{ }^{\circ} \mathrm{C}$ 


\section{UPS analysis}
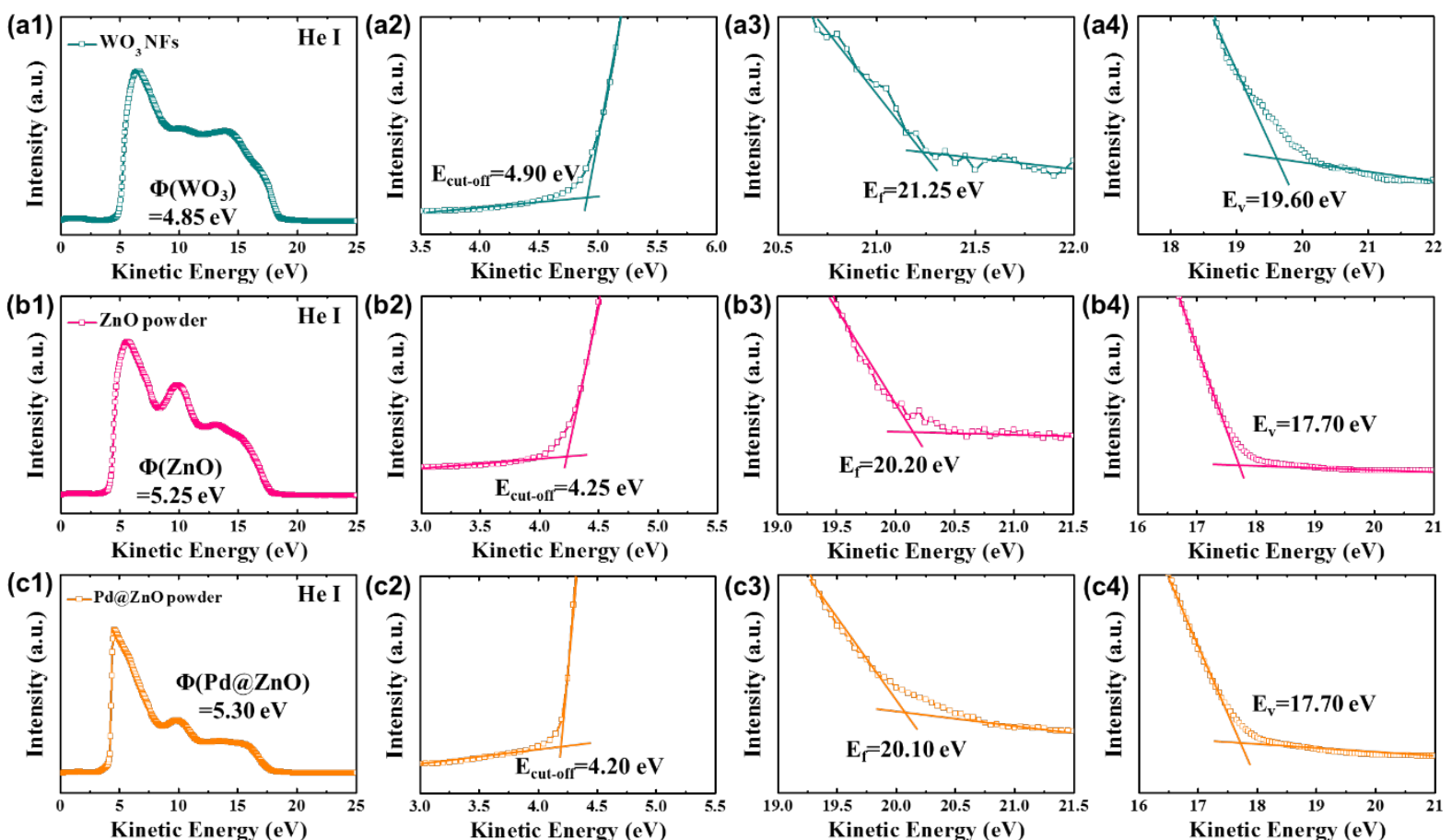

Figure S10. UPS spectra (UV excitation by $\mathrm{He} \mathrm{I}=21.2 \mathrm{eV}$ ) of (a1-a4) $\mathrm{WO}_{3} \mathrm{NFs}$, (b1-b4) $\mathrm{ZnO}$ powders, and (c1-c4) Pd@ZnO powders.

To investigate the work function of samples, we conducted UPS analysis for $\mathrm{WO}_{3} \mathrm{NFs}$, ZnO powders, and Pd@ZnO powders. The work function of samples can be calculated by using the following equation.

$\Phi($ work function $)=h v-\left|\mathrm{E}_{\text {cut }- \text { off }}-\mathrm{E}_{\mathrm{f}}\right|$

The energy source in the UPS analysis was He I $(21.2 \mathrm{eV})$. Therefore, the derived work functions of the $\mathrm{WO}_{3} \mathrm{NFs}$, ZnO powders, and Pd@ZnO powders are 4.85 eV, $5.25 \mathrm{eV}$, and $5.30 \mathrm{eV}$, respectively. 


\section{2. $\mathrm{N}_{2}$ adsorption/desorption and pore size distribution}

(a)

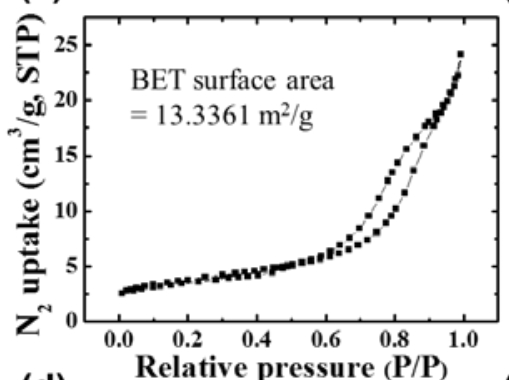

(d)

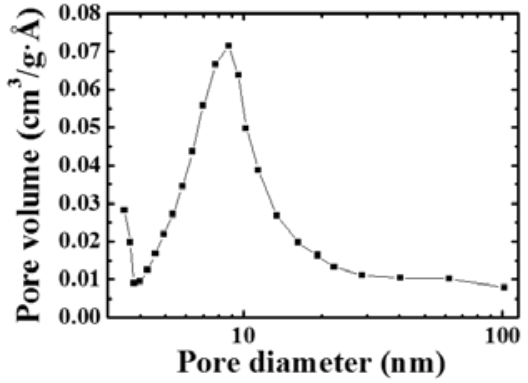

(b)

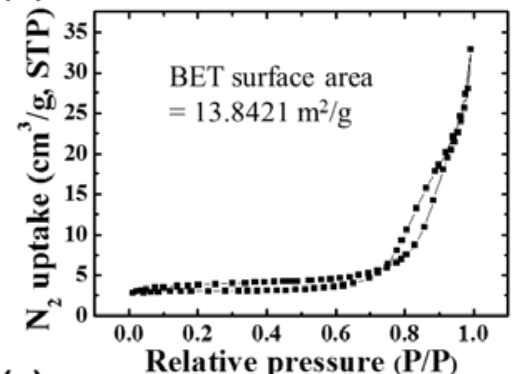

(e)

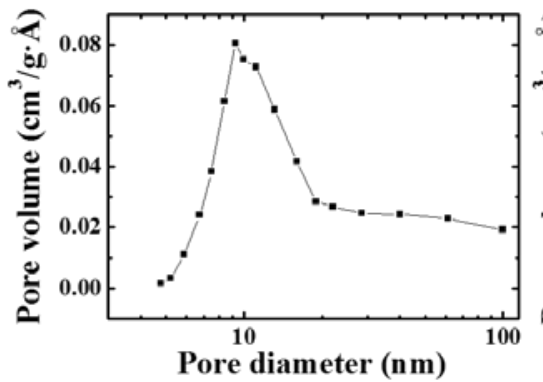

(c)

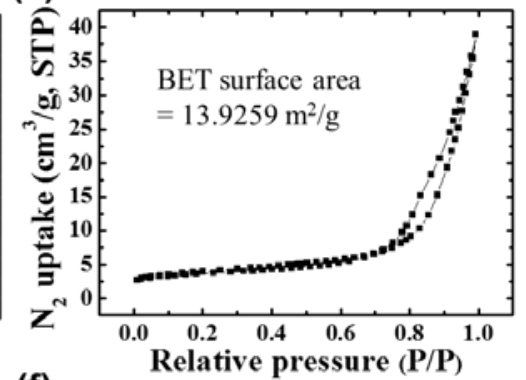

(f)

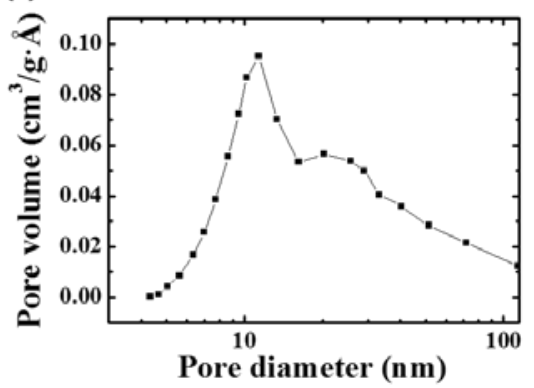

Figure S11. $\mathrm{N}_{2}$ adsorption/desorption isotherms of (a) Pristine $\mathrm{WO}_{3} \mathrm{NFs}$, (b) $\mathrm{ZnO}-\mathrm{WO}_{3} \mathrm{NFs}$, and (c)

$\mathrm{Pd} @ \mathrm{ZnO}-\mathrm{WO}_{3} \mathrm{NFs}$, and pore size distribution of (d) Pristine $\mathrm{WO}_{3} \mathrm{NFs}$, (e) $\mathrm{ZnO}-\mathrm{WO}_{3} \mathrm{NFs}$, and (f) Pd@ZnO- $\mathrm{WO}_{3} \mathrm{NFs}$. 


\section{Supporting table}

Table S1. Recent publications on chemiresistive sensors for detecting toluene gas in various humidity.

\begin{tabular}{|c|c|c|c|c|c|c|c|}
\hline $\begin{array}{c}\text { Gas } \\
\text { species }\end{array}$ & Materials & $\begin{array}{c}\text { Optimal } \\
\text { temperature }\end{array}$ & $\begin{array}{l}\text { Relative } \\
\text { humidity }\end{array}$ & Response & $\begin{array}{c}\text { Response } \\
\text { time }\end{array}$ & $\begin{array}{c}\text { Detection } \\
\text { limit }\end{array}$ & reference \\
\hline \multirow{11}{*}{ Toluene } & $\mathrm{Pd}-\mathrm{WO}_{3} \mathrm{NFs}$ & $350^{\circ} \mathrm{C}$ & $90 \% \mathrm{RH}$ & $\begin{array}{l}5.5 \text { at } \\
1 \mathrm{ppm}\end{array}$ & $10.9 \mathrm{sec}$ & $\begin{array}{l}1.71 \text { at } \\
120 \mathrm{ppb}\end{array}$ & 1 \\
\hline & $\begin{array}{c}\text { Pd-Ps- } \\
\mathrm{WO}_{3} \mathrm{NFs}^{-}\end{array}$ & $350^{\circ} \mathrm{C}$ & $90 \% \mathrm{RH}$ & $\begin{array}{l}12.1 \text { at } \\
5 \mathrm{ppm}\end{array}$ & $28 \mathrm{sec}$ & $\begin{array}{l}2.4 \text { at } \\
100 \mathrm{ppb}\end{array}$ & 2 \\
\hline & $\begin{array}{c}\text { Pt-impregnated } \\
\mathrm{WO}_{3}\end{array}$ & $300^{\circ} \mathrm{C}$ & $90 \% \mathrm{RH}$ & $\begin{array}{l}1.67 \text { at } \\
2 \mathrm{ppm}\end{array}$ & - & - & 3 \\
\hline & $\mathrm{SnO}_{2} \mathrm{NFs}$ & $350^{\circ} \mathrm{C}$ & $25 \% \mathrm{RH}$ & $\begin{array}{c}6.0 \text { at } \\
100 \mathrm{ppm}\end{array}$ & - & $\begin{array}{c}2.0 \text { at } \\
10 \mathrm{ppm}\end{array}$ & 4 \\
\hline & $\mathrm{NiO}-\mathrm{SnO}_{2} \mathrm{NFs}$ & $330^{\circ} \mathrm{C}$ & $25 \% \mathrm{RH}$ & $\begin{array}{c}11 \text { at } \\
50 \mathrm{ppm}\end{array}$ & - & - & 5 \\
\hline & $\begin{array}{l}\mathrm{Co}_{3} \mathrm{O}_{4} \text { hollow } \\
\text { nanospheres }\end{array}$ & $100^{\circ} \mathrm{C}$ & $\begin{array}{c}40-50 \% \\
\text { RH }\end{array}$ & $\begin{array}{c}7 \text { at } \\
200 \mathrm{ppm}\end{array}$ & - & $\begin{array}{c}3 \text { at } \\
10 \mathrm{ppm}\end{array}$ & 6 \\
\hline & $\begin{array}{c}\alpha-\mathrm{Fe}_{2} \mathrm{O}_{3} / \mathrm{SnO}_{2} \\
\text { nanowires arrays }\end{array}$ & $90{ }^{\circ} \mathrm{C}$ & Dry & $\begin{array}{l}1.497 \text { at } \\
100 \mathrm{ppm}\end{array}$ & - & $\begin{array}{l}1.25 \text { at } \\
50 \mathrm{ppm}\end{array}$ & 7 \\
\hline & $\begin{array}{l}\mathrm{Au}-\mathrm{ZnO} \\
\text { nanowire }\end{array}$ & $340^{\circ} \mathrm{C}$ & Dry & $\begin{array}{l}6.275 \text { at } \\
10 \mathrm{ppm}\end{array}$ & $50 \mathrm{sec}$ & $\begin{array}{l}2.3 \text { at } \\
1 \mathrm{ppm}\end{array}$ & 8 \\
\hline & $\mathrm{TiO}_{2}$ doped $\mathrm{ZnO}$ & $290^{\circ} \mathrm{C}$ & Dry & $\begin{array}{c}10.9 \text { at } \\
100 \mathrm{ppm}\end{array}$ & $8.0 \mathrm{sec}$ & $\begin{array}{l}1.9 \text { at } \\
1 \mathrm{ppm}\end{array}$ & 9 \\
\hline & $\mathrm{ZnO}-\mathrm{SnO}_{2} \mathrm{NFs}$ & $360^{\circ} \mathrm{C}$ & Dry & $\begin{array}{c}9.8 \text { at } \\
100 \mathrm{ppm}\end{array}$ & - & - & 10 \\
\hline & $\begin{array}{l}\mathrm{Pd@ZnO-} \\
\mathrm{WO}_{3} \mathrm{NFs}\end{array}$ & $350^{\circ} \mathrm{C}$ & $90 \% \mathrm{RH}$ & $\begin{array}{c}22.22 \\
\text { at } 1 \mathrm{ppm}\end{array}$ & $8.0 \mathrm{sec}$ & $\begin{array}{l}4.37 \text { at } \\
100 \mathrm{ppb}\end{array}$ & this work \\
\hline
\end{tabular}




\section{Supporting references}

\section{References}

(1) Kim, N. H.; Choi, S. J.; Yang, D. J.; Bae, J.; Park, J.; Kim, I. D., Sensor Actuat. B 2014, 193, 574.

(2) Choi, S. J.; Kim, S. J.; Koo, W. T.; Cho, H. J.; Kim, I. D., Chem. Commun. 2015, 51 (13), 2609.

(3) Lee, I.; Choi, S. J.; Park, K. M.; Lee, S. S.; Choi, S.; Kim, I. D.; Park, C. O., Sensor Actuat. B 2014, 197, 300.

(4) Qia, Q.; Zhang, T.; Liu, L.; Zheng, X. J., Sensor Actuat. B 2009, 137, 471.

(5) Liu, L.; Zhang, Y.; Wang, G. G.; Li, S. C.; Wang, L. Y.; Han, Y.; Jiang, X. X.; Wei, A. G., Sensor Actuat. B 2011, 160, 448.

(6) Park, J.; Shen, X.; Wang, G. Sensor Actuat. B 2009, 136, 494.

(7) Wang, T. S.; Huang, Z. S.; Yu, Z. D.; Wang, B.; Wang, H.; Sun, P.; Suo, H.; Gao, Y.; Sun, Y. F.; Li, T.; Lu, G., RSC Adv. 2016 6, 52604.

(8) Wang, L. W.; Wang, S. R.; Xu, M. J.; Hu, X. J.; Zhang, H. X.; Wang, Y. S.; Huang, W. P., Phys. Chem. Chem. Phys. 2013, 15, 17179.

(9) Zeng, Y.; Zhang, T.; Wang, L. J.; Kang, M. H.; Fan, H. T.; Wang, R.; He, Y., Sensor Actuat. B-Chem 2009, 140, 73.

(10) Song, X. F.; Zhang, D. J.; Fan, M., Appl. Surf. Sci. 2009, 255, 7343. 